\title{
Real Time Demonstration of the Transport of Ethernet Fronthaul based on vRAN in Optical Access Networks
}

\author{
Z. Tayq ${ }^{(1,2)}$, L. Anet Neto ${ }^{(1)}$, B. Le Guyader ${ }^{(1}$, A. De Lannoy ${ }^{(1)}$, M. Chouaref ${ }^{(1)}$, C. Aupetit-Berthelemot $^{(2)}$, \\ M. Nelamangala Anjanappa ${ }^{(3)}$, S. Nguyen ${ }^{(3)}$, K. Chowdhury $^{(3)}$, P. Chanclou ${ }^{(1)}$ \\ ${ }^{\prime}$ Orange Labs Networks, 2 Avenue P.Marzin, 22300 Lannion, France ${ }^{2}$ Laboratoire XLIM, 16 rue d'Atlantis, 87068 Limoges CEDEX, France, \\ ${ }^{3}$ Altiostar, 200 Ames Pond Dr, Tewksbury, MA 01876, USA \\ zakaria.tayq@orange.com
}

\begin{abstract}
A real-time transmission of a new functional split fronthaul interface over PtP and PtMP optical access networks is experimentally demonstrated. The data traffic evolution is investigated as well as the impact of latency and packet loss.

OCIS codes: (060.0060) Fiber optics and optical communications; (060.4250) Networks
\end{abstract}

\section{Introduction}

Passive Optical Network (PON) technologies are considered as the most attractive support of the present and future fixed broadband fiber access. Optical fiber is also considered as a much-needed medium to support mobile access networks. With the rapid development of mobile internet services, operators are facing challenges to cope with the data traffic increase which obliges them to use optical access infrastructure and technologies in their Radio Access Networks (RANs). Currently, optical fiber is commonly used to achieve the mobile Ethernet backhaul between the base station sites and the core network. Fronthaul is another emerging network segment considered in RAN evolution. It concerns the connection from the Base Band Unit (BBU) to the Remote Radio Head (RRH) in a Cloud RAN (C-RAN) context [1]. In this case, fronthaul carries the very high bit rate digitized radio signals, according in most cases to the Common Public Radio Interface (CPRI) [1].

Because of the very high capacities required for the $5 \mathrm{G}$, other transport approaches are now being considered by the mobile community following the trends of RAN virtualization. In fact, lots of initiatives exist presently to define new functional splits between BBU and RRH [2] [3]. Their main objective is to migrate, at a higher or lower proportion, some signal processing blocks from the BBU to the RRH in order to allow bit-rate reduction in the fronthaul while ensuring proper CoMP (Coordinated MultiPoint). This will also permit the BBU virtualization since the high layer functions of LTE don't have real time constraints, therefore generic servers could be used to host these functions. A basic illustrative concept of RAN block diagram is show in Figure 1.a. The technical drivers of this new fronthaul, expected to be Ethernet packet based, are mainly: decrease the line rate, relax latency constrains, allow compatibility with the statistical multiplexing and enable the re-use of the existing Ethernet backhaul network. Figure 1.b illustrates the new RAN architecture based on Ethernet fronthaul. The BBUs, currently located at the antenna site, are virtualized and moved to the Master $\mathrm{CO}$ allowing resource pooling and reusing the existing backhaul, including access and aggregation segments. Despite the more relaxed bit-rate constraints allowed by functional split solutions, deporting high-layer LTE functions to the Master CO will still need higher throughputs compared to regular backhaul. This is why, it is vital to evaluate the capacity of the existing infrastructure to handle such traffic. Moreover, the transport equipment brings latency and packet loss in the network, the robustness of the new fronthaul interface towards these impairments must also be assessed. In this paper, we focus on the functional split at the Packet Data Convergence Protocol (PDCP) level and investigated in real time the previously exposed concerns about the transported data traffic and the impact of impairments on the new fronthaul interface. The transmission is done over Point-to-Point (PtP) and Point to MultiPoint (PtMP) optical access networks.

a)

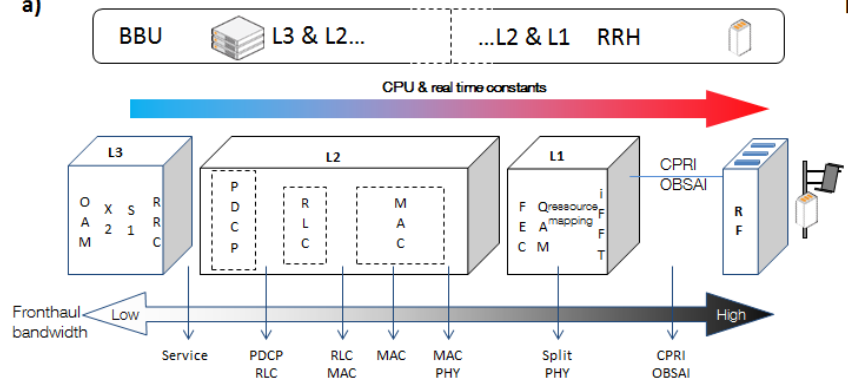

Figure 1: a) Functional Splits in the RAN equipment b)

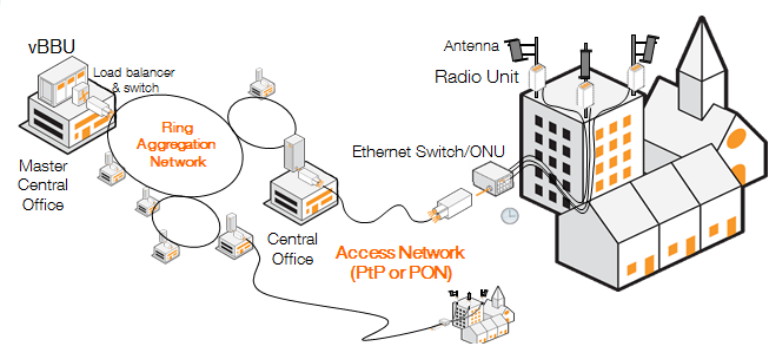

b) Illustrative architecture of new Ethernet fronthaul 


\section{Experimental setup:}

As shown in figure 2, we experimentally investigated two Ethernet-based solutions for the transport of the new fronthaul interface: Point-to-Point and Point-to-Multipoint with a G-PON (Gigabit capable PON) system.

At the transmitter side, generic servers running on OpenStack host a virtual EMS (Element Management System), a virtual EPC (Evolved Packet Core) where the Mobile Edge Computing (MEC) is happening and a virtual BBU (vBBU). A 10G Top of Rack (ToR) Ethernet switch is used to connect these nodes. At the receiver side, an intelligent RRH (iRRH) is connected to a User Equipement (UE) (here, a computer) via a mobile dongle. We use a mobile signal based on a LTE (Long Term Evolution) with $15 \mathrm{MHz}$ bandwidth and 2x2 MIMO (Multiple Input Multiple Output). The synchronization in this network is provided by Global Positioning System (GPS) devices connected to each iRRH.

For PtP fronthaul, we used common $1 \mathrm{Gbit} / \mathrm{s}$ Ethernet switches in both ends. Packet loss and latency are then inserted with a dedicated test device in order to evaluate its impact on the mobile throughput.

For the PtMP fronthaul, an Optical Line Termination (OLT) is installed after the ToR switch and an Optical Network Unit (ONU) is associated to each iRRH. We allocated fixed bandwidths for the upstream to the ONUs, $200 \mathrm{Mb} / \mathrm{s}$ for ONU 1 connected to the iRRH 2 and $600 \mathrm{Mb} / \mathrm{s}$ for ONU 2 used for overloading the G-PON system. With this configuration, we evaluated the LTE throughput for different lengths of optical fiber.

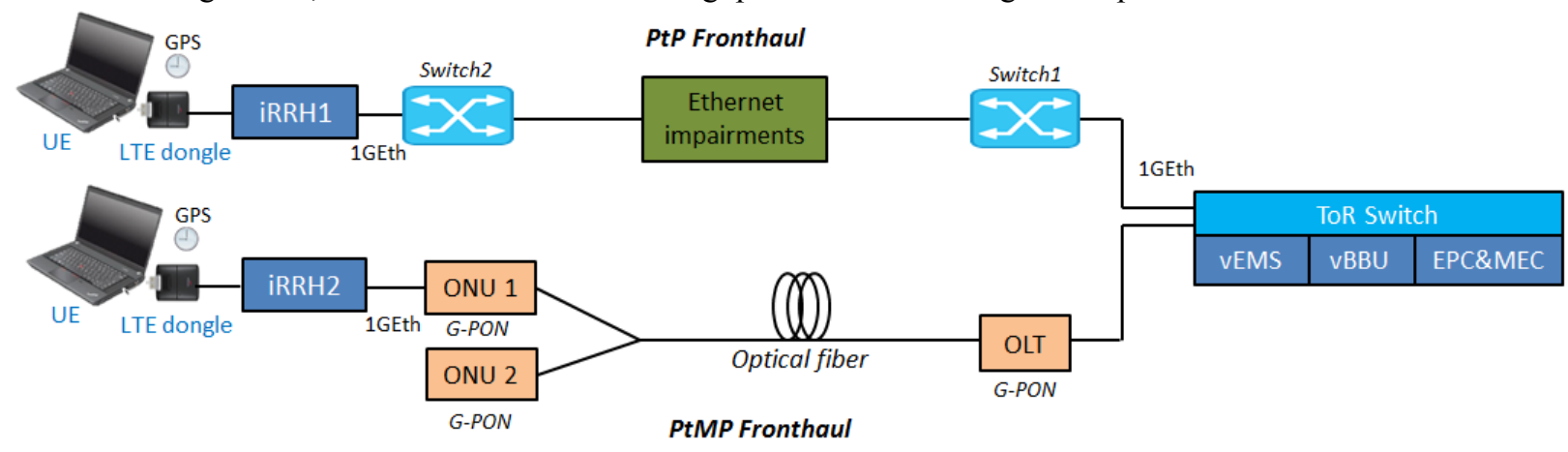

Figure 2: Experimental setup

\section{Experimental results discussion:}

We started our investigation with the PtP configuration. Figure 3.a depicts the impact of the used functional slit on the traffic of the vRAN. For the downstream, we generated traffic with different bit rates in the EPC and evaluated the bitrate in the backhaul, fronthaul and the UE. In the upstream, we generated traffic in the UE and measured the bit rate in the fronthaul, backhaul and EPC. It is interesting to notice that the traffic in the fronthaul varies depending on the mobile traffic demand unlike the currently used functional split with CPRI, which works at fixed bit-rates (here, CPRI3 corresponding to $2.5 \mathrm{Gbit} / \mathrm{s}$ in this radio configuration). In the downlink (DL), we observe an increase of 5\% in the backhaul bitrate compared to the generated traffic in the EPC, which is due to the encapsulation in Ethernet frames. A $20 \%$ increase of traffic is measured in the fronthaul compared to the backhaul. This traffic eventually stabilizes when the maximum radio throughput is reached because the vBBU adapts the fronthaul bit-rate to the available radio resources. A similar behaviour is observed in the uplink (UL).

Figure 3.b shows the impact of packet loss on the mobile LTE throughput in the downstream and upstream using TCP (Transmission Control Protocol) and UDP (User Datagram Protocol) for the mobile signal between EPC and UE. In few words, TCP continuously adapts the transmission bit-rate to the network capacity to avoid congestion while UDP transmits at a constant bit-rate. We can see that the LTE throughput is not impacted by packet loss down $1 \%$ for UDP transmission. For TCP, the impact on throughput is negligible for packet loss down $0.1 \%$ for the downstream and $0.4 \%$ for the upstream. Therefore, the transmission is robust since only $10^{-3}$ packet loss for nonpriority data and $10^{-5}$ for priority data is recommended generally for Ethernet transmissions in the backhaul. Throughput degradation is noticed between TCP DL and UDP DL at 0\% packet loss, which is due to the limited buffer capacity of the used LTE dongle. Ulterior tests with another dongle show similar TCP and UDP throughput at $0 \%$ packet loss. The one way latency impact is represented in figure 3.c. No impact is observed for injected latencies down to $20 \mathrm{~ms}$. Knowing that the latency in the current LTE network, between the master CO and the cell site gateway (Figure1.b), is between 5 and $10 \mathrm{~ms}$, the re-use of the existing network for fronthaul should not affect the performances. Regardless, these values must be reduced for the ultra-low latency applications expected in $5 \mathrm{G}$. 

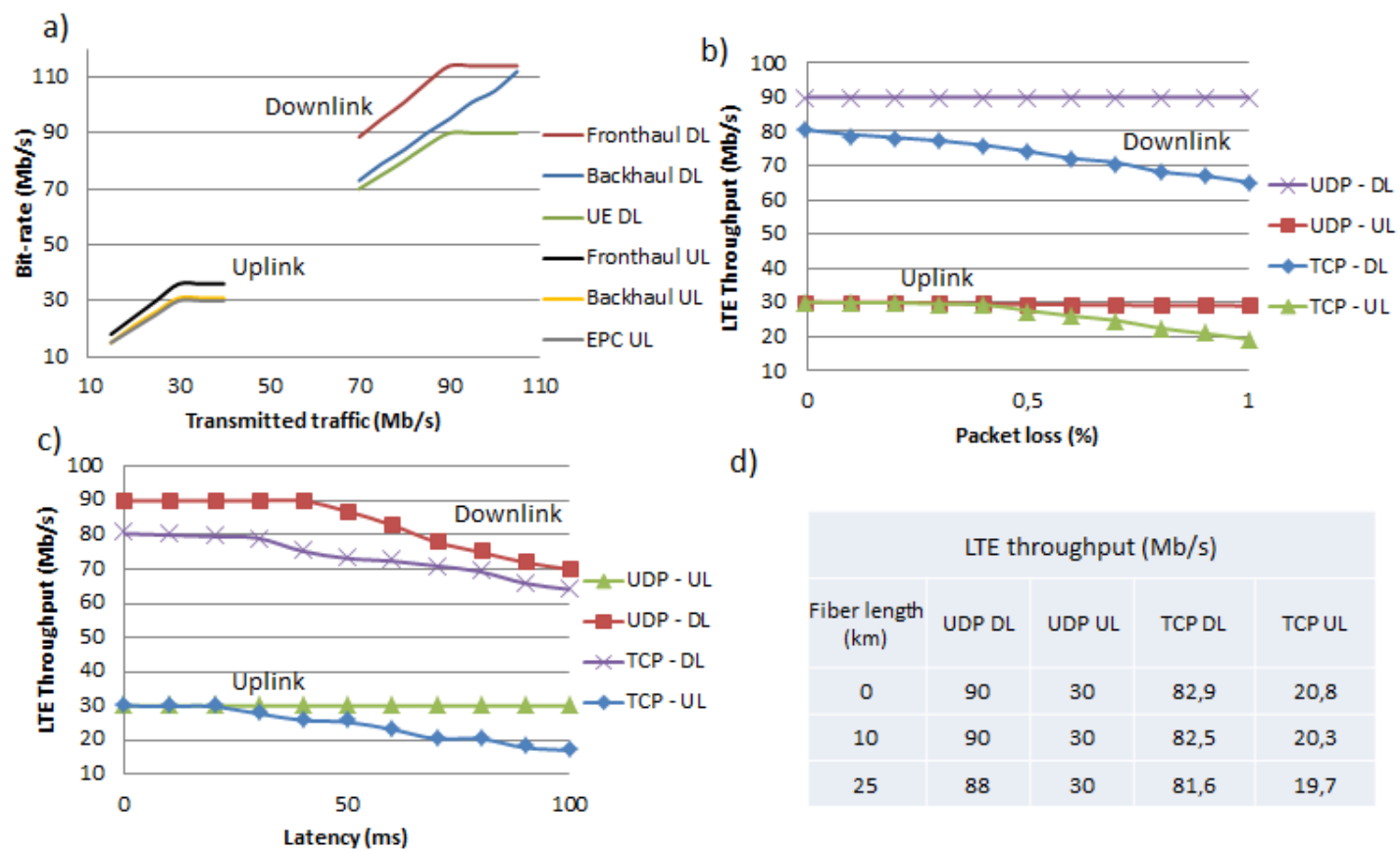

d)

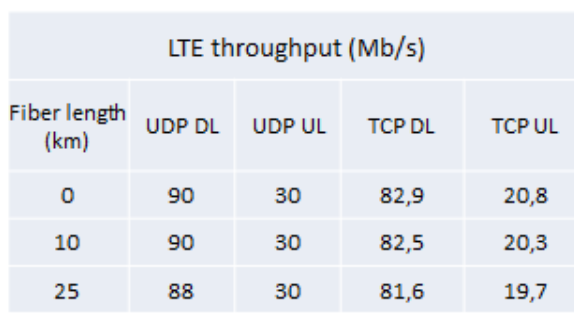

Figure 3: (a) xhaul traffic in the downlink and uplink (b) Packet loss (\%) vs LTE Throughput (Mb/s) (c) Latency (ms) vs LTE Throughput (Mb/s) (d) Mobile throughput downlink and uplink for fronthaul over G-PON

PtMP architecture based on PON is also an adequate candidate to accommodate fronthaul considering the large availability of Fiber to the Home (FTTH) infrastructures. It was difficult to achieve this with CPRI based fronthaul because of bandwidth and latency issues [4]. Since these metrics were relaxed in the proposed solution, G-PON can be used for the access part of the architecture shown in figure1.b. The Dynamic Bandwidth Allocation (DBA) algorithm based on status reporting method introduces around $1 \mathrm{~ms}$ latency [4], which is too much for the access considering $5 \mathrm{G}$ requirements. One approach to reduce the DBA latency is to use a fixed bandwidth allocation scheme as described in the experimental setup. Figure 3.d shows the results for fronthaul over G-PON. No impact is noticed when overloading the system with a second ONU (named ONU 2 in figure 2) using an Ethernet tester. In fact, for the downstream, we obtained performances similar to PtP for $0 \mathrm{~km}$ and $10 \mathrm{~km}$. A little impact is noticed in $25 \mathrm{~km}$ since it's the maximal distance allowed with the used G-PON system. For TCP transmission in the uplink, we notice throughput degradation compared to PtP. This could be due to the packet latency unbalance ( $40 \mu \mathrm{s}$ in the downstream and $140 \mu \mathrm{s}$ in the upstream) and/or the packet jitter $(0.9 \mu \mathrm{s}$ in the upstream and $48 \mu \mathrm{s}$ in the upstream) introduced by the G-PON system, which affects the queuing policies used by the vRAN equipment. Some adjustments based on coordination between BBU and OLT are then needed to transport this new functional split fronthaul with no impact on the LTE throughput and the quality of the delivered services [5].

\section{Conclusions}

In this paper, we demonstrated in real time the transport of a new fronthaul interface based on a new functional split over an Ethernet network based on PtP switch and over a G-PON system. The results show $20 \%$ increase of traffic compared to regular backhaul. Also throughput measurements show potentially that backhaul and FTTH infrastructures can be used for the transport of this Ethernet Fronthaul with the necessity to do some joint adjustments in the RAN and transport equipment (queuing policies...).

\section{Acknowledgements}

This work has been funded by the European Union's Horizon 2020 iCirrus project (grand no. 644526) and 5G-PPP 5G-Crosshaul (grant no. 671598).

\section{References}

[1] A. Pizzinat, P. Chanclou, F.Saliou, T.Diallo, "Things You Should Know About Fronthaul“, JLT, (2015)

[2] "Small Cell Virtualization: Functionnal Splits and Use cases" Small Cell Forum release 6, 2016

[3] White Paper of Next Generation Fronthaul Interface, version 1.0, October 4, 2015

[4] J. Terada,T. Shimada, T. Shimizu, A. Otaka , "Optical Network Technologies for Wireless Communication Network" , ECOC 2016

[5] J-I Kani, J. Terada, K-I Suzuki, A. Otaka, "Solutions for future mobile fronthaul and access-network convergence", JLT (2016) 